\title{
The interplay between apoptosis and ferroptosis mediated by ER stress
}

\author{
Yong J. Lee ${ }^{1} \mathbb{D}$
}

Accepted: 25 October 2020 / Published online: 2 November 2020

○) Springer Science+Business Media, LLC, part of Springer Nature 2020

\section{Reply}

In our recent article [1], we report that ER stress response plays a pivotal role in the crosstalk between ferroptosis and apoptosis. Dr. Yoshida constructively criticizes that TRAIL treatment alone leads to JC-1 aggregation and combined treatment of TRAIL and erastin results in mitochondrial depolarization which is indicated by an increase in JC-1 fluorescence intensity. Since we used a sublethal dose of TRAIL, the degree of JC-1 aggregation didn't significantly change during treatment with TRAIL alone (Fig. 4C). However, the combined treatment led to significant increasd membrane permeability (loss of mitochondrial membrane potential) and consequently less amount of JC-1 aggregates in the mitochondria. Thus, under this promoted apoptotic death condition, we observed an increase in JC-1 fluorescence intensity (retaining its origical green fluorescence) [2].

Moreover, Dr. Yoshida suggests several possible mechanisms to maintain the balance between pro-apoptotic PUMA protein and anti-apoptotic Bcl-2 family proteins to prevent apoptosis during treatment with ferroptotic agent erastin. We agree with Dr. Yoshida' comment. It is possible that imbalance between them occurs during the combined treatment and results in promoting apoptosis. The imbalance is probably due to the reduction of Bcl-2 family proteins through phosphorylated JNK-mediated phosphorylation and subsequent ubiquitination during the combined treatment $[3,4]$. We also agree with Dr. Yoshida's comment. It is possible that Beclin-1 is a key molecule in the crosstalk between ferroptosis and apoptosis. Akt-mediated dephosphorylation of Beclin-1 at Ser 234/295 may occur and enhace Beclin-1 cleavage and enhance apoptosis during the

combined treatment [5]. Obviously, these possibilities need to be further examined to understand the role of PUMA in the combinatorial treatment-induced synergistic apoptosis.

Funding Funding was supported by Grant Nos. NCI R03CA205267, NCI R03CA212125, DOD W81XWH-20-1-0190.

\section{Compliance with ethical standards}

Conflict of interest The author declares no competing financial interests.

\section{References}

1 Lee YS, Kalimuthu K, Park YS et al (2020) BAX-dependent mitochondrial pathway mediates the crosstalk between ferroptosis and apoptosis. Apoptosis. https://doi.org/10.1007/s10495-020-01627 $-\mathrm{z}$

2. Sivandzade F, Bhalerao A, Cucullo L (2019) Analysis of the mitochondrial membrane potential using the cationic JC-1 dye as a sensitive fluorescent probe. Bio Protoc 9:e3128

3. Inoshita S, Takeda K, Hatai T et al (2002) Phosphorylation and inactivation of myeloid cell leukemia 1 by JNK in response to oxidative stress. J Biol Chem 277:43730-43734

4. Inuzuka H, Shaik S, Onoyama I et al (2011) SCF (FBW7) regulates cellular apoptosis by targeting MCL1 for ubiquitylation and destruction. Nature 471:104-109

5. Wirawan E, Vande Walle L, Kersse K (2010) Caspase-mediated cleavage of Beclin-1 inactivates Beclin-1-induced autophagy and enhances apoptosis by promoting the release of proapoptotic factors from mitochondria. Cell Death Dis 1:e18

Publisher's Note Springer Nature remains neutral with regard to jurisdictional claims in published maps and institutional affiliations.

Yong J. Lee

leeyj@upmc.edu

1 Department of Surgery, School of Medicine, University of Pittsburgh, 5117 Centre Ave, Pittsburgh, PA 15213, USA 